\title{
Interaction study of Pasteurella multocida with culturable aerobic bacteria isolated from porcine respiratory tracts using coculture in conditioned media
}

\author{
Nonzee Hanchanachai ${ }^{1,2}$, Pramote Chumnanpuen ${ }^{2,3}$ and Teerasak E-kobon ${ }^{2,4,5^{*}}$ (D)
}

\begin{abstract}
Background: The porcine respiratory tract harbours multiple microorganisms, and the interactions between these organisms could be associated with animal health status. Pasteurella multocida is a culturable facultative anaerobic bacterium isolated from healthy and diseased porcine respiratory tracts. The interaction between $P$. multocida and other aerobic commensal bacteria in the porcine respiratory tract is not well understood. This study aimed to determine the interactions between porcine P. multocida capsular serotype A and D strains and other culturable aerobic bacteria isolated from porcine respiratory tracts using a coculture assay in conditioned media followed by calculation of the growth rates and interaction parameters.
\end{abstract}

Results: One hundred and sixteen bacterial samples were isolated from five porcine respiratory tracts, and 93 isolates were identified and phylogenetically classified into fourteen genera based on $16 \mathrm{~S}$ rRNA sequences. Thirteen isolates from Gram-negative bacterial genera and two isolates from the Gram-positive bacterial genus were selected for coculture with P. multocida. From $17 \times 17$ (289) interaction pairs, the majority of 220 pairs had negative interactions indicating competition for nutrients and space, while 17 pairs were identified as mild cooperative or positive interactions indicating their coexistence. All conditioned media, except those of Acinetobacter, could inhibit P. multocida growth. Conversely, the conditioned media of $P$. multocida also inhibited the growth of nine isolates plus themselves.

Conclusion: Negative interaction was the major interactions among the coculture of these 15 representative isolates and the coculture with $P$. multocida. The conditioned media in this study might be further analysed to identify critical molecules and examined by the in vivo experiments. The study proposed the possibility of using these molecules in conditioned media to control P. multocida growth.

Keywords: Pasteurella multocida, Bacteria-bacteria interaction, Coculture, Porcine respiratory tract, Conditioned media

\footnotetext{
* Correspondence: teerasak.e@ku.th

${ }^{2}$ Computational Biomodelling Laboratory for Agricultural Science and

Technology, Kasetsart University, Bangkok 10900, Thailand

${ }^{4}$ Department of Genetics, Faculty of Science, Kasetsart University, Bangkok

10900, Thailand

Full list of author information is available at the end of the article
}

(c) The Author(s). 2021 Open Access This article is licensed under a Creative Commons Attribution 4.0 International License, which permits use, sharing, adaptation, distribution and reproduction in any medium or format, as long as you give appropriate credit to the original author(s) and the source, provide a link to the Creative Commons licence, and indicate if changes were made. The images or other third party material in this article are included in the article's Creative Commons licence, unless indicated otherwise in a credit line to the material. If material is not included in the article's Creative Commons licence and your intended use is not permitted by statutory regulation or exceeds the permitted use, you will need to obtain permission directly from the copyright holder. To view a copy of this licence, visit http://creativecommons.org/licenses/by/4.0/ The Creative Commons Public Domain Dedication waiver (http://creativecommons.org/publicdomain/zero/1.0/) applies to the data made available in this article, unless otherwise stated in a credit line to the data. 


\section{Background}

The porcine respiratory system is exposed to external environments and foreign particles, including bacteria, viruses and pollutants, through inhalation and exhalation processes [1,2]. Respiratory diseases are associated with economic loss in the swine industry [3, 4]. Metagenomic studies revealed that several bacteria predominantly colonized the porcine respiratory tract, including those in the phyla Firmicutes, Proteobacteria, and Bacteroidetes, and changes in these bacteria were associated with porcine health status [5-8]. Piglets with Glässer's disease in Spain had a higher number of Proteobacteria in the families Pasteurellaceae and Moraxellacea and lower number of Firmicutes in the Ruminococcaceae family in the nasal cavity compared to the healthy piglets $[5,7]$. Huang et al. [8] used 16S rRNA metagenomic sequencing to examine 20 swine lungs. They found that the healthy lungs prevalently had bacteria from the genera Methylotenera, Prevotella, Sphingobium, and Lactobacillus, whereas the genera Mycoplasma, Ureaplasma, Sphingobium, Haemophilus, and Phyllobacterium were abundant in the severe-lesion lungs. The microbial diversity inside these lesion lungs decreased when the population of certain bacteria increased. These studies have raised questions on how these porcine respiratorytract bacteria interact and control the balance of commensal and pathogenic bacteria in the community.

A member of the Pasteurellaceae family, Pasteurella multocida, commonly inhabits the nasopharynx of birds and mammals and can be associated with economically significant diseases in pigs, including progressive atrophic rhinitis (PAR) caused by the toxin-producing strains, pneumonic pasteurellosis, and porcine respiratory disease complex (PRDC) caused by multiple pathogens [9-11]. The porcine strains of $P$. multocida commonly belong to capsular types A and D, which could be isolated from the nose, tonsils and upper respiratory tract of both healthy and diseased pigs [12-15]. The non-toxigenic capsular type A strains could be the primary agent of pneumonia and septicemia in 100-day-old pigs [12,16] and caused dermatitis and nephropathy syndrome (PNDS) in growing and finishing pigs $[12,16]$. Several in vitro and in vivo bacteria-bacteria interaction studies focused on the interaction between $P$. multocida and other primary respiratory pathogens in the pathogenic process. The porcine toxigenic capsular type A and D strains of $P$. multocida can be primary pathogens or coinfect piglets with Bordetella bronchiseptica, causing PAR under stress and immunocompromised conditions [17]. Colonization of these strains in porcine tracheal rings increased during co-infection with $B$. bronchiseptica [18]. The adherence study of $P$. multocida and B. bronchiseptica to swine nasal epithelial cells found that $P$. multocida could not colonize the swine nasal mucosa well compared to $B$. bronchiseptica [19, 20]. The number of B. bronchiseptica cells adhered to the nasal epithelial cells was three times higher than the number of $P$. multocida cells, suggesting the opportunistic role of $P$. multocida after B. bronchiseptica infection $[19,20]$. The co-infection of $P$. multocida with other pathogens could enhance disease damage to the hosts; e.g., promote secure attachment of the bacteria to the bovine respiratory syncytial virus-infected cells [21] and increase inflammatory cells in the coinfected lesions of bronchopneumonia pigs [22]. Recently, Bartkiene et al. developed a combination of a plant extract with a probiotic bacterium Lactobacillus uvarum that could inhibit $P$. multocida growth in vitro [23]. This finding has led to hypothesize the role of the porcine respiratory tract normal flora on P. multocida growth. However, the interactions of P. multocida with the commensal bacteria in the porcine respiratory tract are less understood.

In vitro and in vivo coculture assays are frequently employed to study bacterial interactions. Different bacterial species could be directly cocultured together by the planktonic mixed culture [24-26] with or without physical separation [27] and the host cell model [28]. Indirect coculture could be another option by growing one bacterial species in the culture medium used by another species, also called spent or conditioned medium [29]. De Vos et al. [29] examined the polymicrobial interaction of 72 bacterial samples isolated from 23 individuals with urinary tract infections by using a coculture assay in the spent media. The study found that competitive $(-/-)$ and cooperative $(+/+)$ interactions were more common than exploitive interactions $(+/-)$ and that competitive interactions were enriched among individuals. As explained in the above example, bacterial culture in the conditioned media could be easily monitored and expanded to observe responses by comparing to the control culture. As the interaction studies between $P$. multocida and other commensal bacteria in the porcine respiratory tract remain not well understood, this study aimed to initially determine the in vitro interactions between the porcine capsular type A and D strains of $P$. multocida and other culturable aerobic bacteria isolated from porcine respiratory tracts using the coculture assay in conditioned media. Understanding these interactions would benefit further examination of the in vivo bacterial interactions in the porcine respiratory tract. It would assist the process of respiratory disease control in improving porcine health and welfare.

\section{Results}

Culturable aerobic bacteria from porcine respiratory tracts

One hundred and sixteen aerobic bacterial isolates from five porcine respiratory tracts were successfully cultured from the trachea, tracheobronchial lymph node, apical lobe, cardiac lobe, and diaphragmatic lobe of both the left and right lungs. An average of $23 \pm 10$ isolates was 
obtained from each lung. The L1 and L4 lungs had the highest numbers of 31 and 36 isolates, respectively. The majority of the isolates (56\%) were from the apical and diaphragmatic lobes of the lungs. Almost $90 \%$ of these isolates were gram-negative rod-shaped bacteria that had different colony characteristics, i.e., colony forms $(63 \%$ circular and 37\% irregular), margins (55\% undulate, 26\% entire, $18 \%$ curled, and $1 \%$ lobate), and mucosity (66\% nonmucoid and 34\% mucoid). Ninety-three (80\%) of these aerobic culturable bacteria were successfully identified and classified into 14 genera and 21 species (Additional file 1) from seven families of three bacterial phyla (97\% Proteobacteria, 17\% Firmicutes, and 2\% Bacteroidetes), i.e., Acidovorax, Acinetobacter, Aeromonas, Escherichia, Enterobacter, Hafnia, Klebsiella, Macrococcus, Proteus, Providencia, Shewanella, Shigella, Weeksella, and Wohlfahrtiimonas, based on $16 \mathrm{~S}$ rRNA sequence analysis (Figs. 1 and 2). The accession numbers of the sequences were shown in Additional file 1. From Fig. 1, the prevalence of the aerobic culturable bacterial isolates in five parts of the porcine respiratory tracts differed. Three genera (Proteus, Acinetobacter, and Klebsiella) were abundant in the trachea (89\%). In comparison, ten genera isolated from the tracheobronchial lymph node were the most diverse $(53 \%)$ with three abundant genera (Aeromonas, Klebsiella, and Macrococcus). Moreover, the three lobes of the porcine lung also showed different abundance: Acinetobacter (43\%) in the cardiac lobe, Escherichia and Proteus (43\%) in the diaphragmatic lobe, and Macrococcus and Proteus (44\%) in the apical lobe. Phylogenetic analysis of the 16S rRNA sequences clustered these 93 isolates into seven major groups (Fig. 2). The first four groups (60\%) were members of the family Enterobacteriaceae, including Escherichia, Shigella, Enterobacter, Klebsiella, Hafnia,
Proteus, and a small cluster of Providencia. The fifth group contained Macrococcus, which was the only gram-positive bacterial genus belonging to the family Staphylococcaceae. The sixth and seventh groups consisted of the genera Acinetobacter (family Moraxellaceae) and Aeromonas (family Aeromonadaceae). The remaining isolates had only one or two members, including Acidovorax (family Comamonadaceae), Shewanella (family Shewanellaceae), Wohlfahrtiimonas (unclassified bacteria in the class Gammaproteobacteria), and Weeksella (family Flavobacteriaceae). One isolate was selected to represent each identified genus, except two isolates of Macrococcus, NS20 (G5) and NS108 (G7), for the only gram-positive bacterial group as displayed in Fig. 2.

Growth of the selected aerobic bacterial isolates from the porcine respiratory tracts in different conditioned media

Fifteen isolates from 14 genera of the isolated aerobic bacteria from the porcine respiratory tracts and two porcine strains of $P$. multocida with capsular types A and D (PM7 and PM2) were cocultured in the conditioned media (spent BHIB) and the unconditioned media (fresh BHIB), resulting in 289 interacting pairs $(17 \times 17)$ as shown in Fig. 3. Nearly all conditioned media could inhibit the growth of these two P. multocida strains (the first two rows of Fig. 3), except that of Acinetobacter. The conditioned medium of Acinetobacter supported or slightly slowed the growth of all tested bacteria. The conditioned medium of Providencia inhibited the growth of every isolate, including itself. The media of Shigella and Macrococcus NS108 (G7) had a lower inhibitory effect on Klebsiella, Escherichia, Shigella, and Enterobacter. Conditioned media from Proteus and Escherichia only supported the growth of Klebsiella

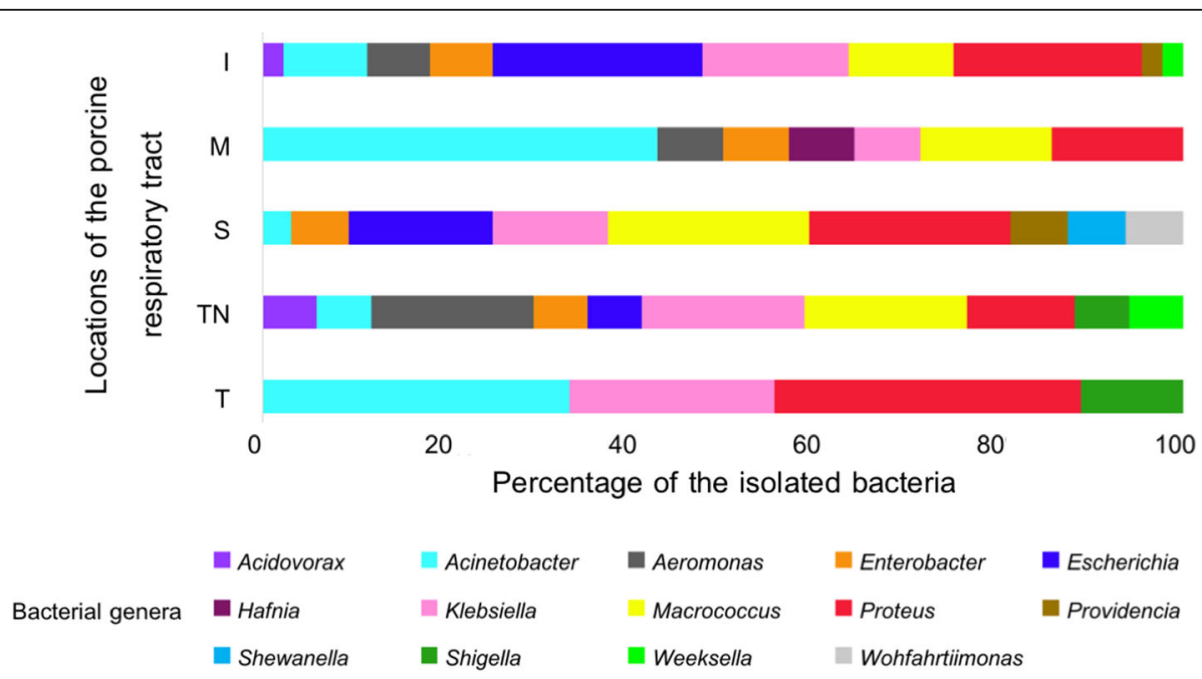

Fig. 1 Percentage of isolated aerobic and culturable bacterial genera from different locations of the porcine respiratory tracts. T; Trachea, TN; Tracheobronchial lymph node, S; Apical lobe, M; Cardiac lobe, and I; Diaphragmatic lobe 


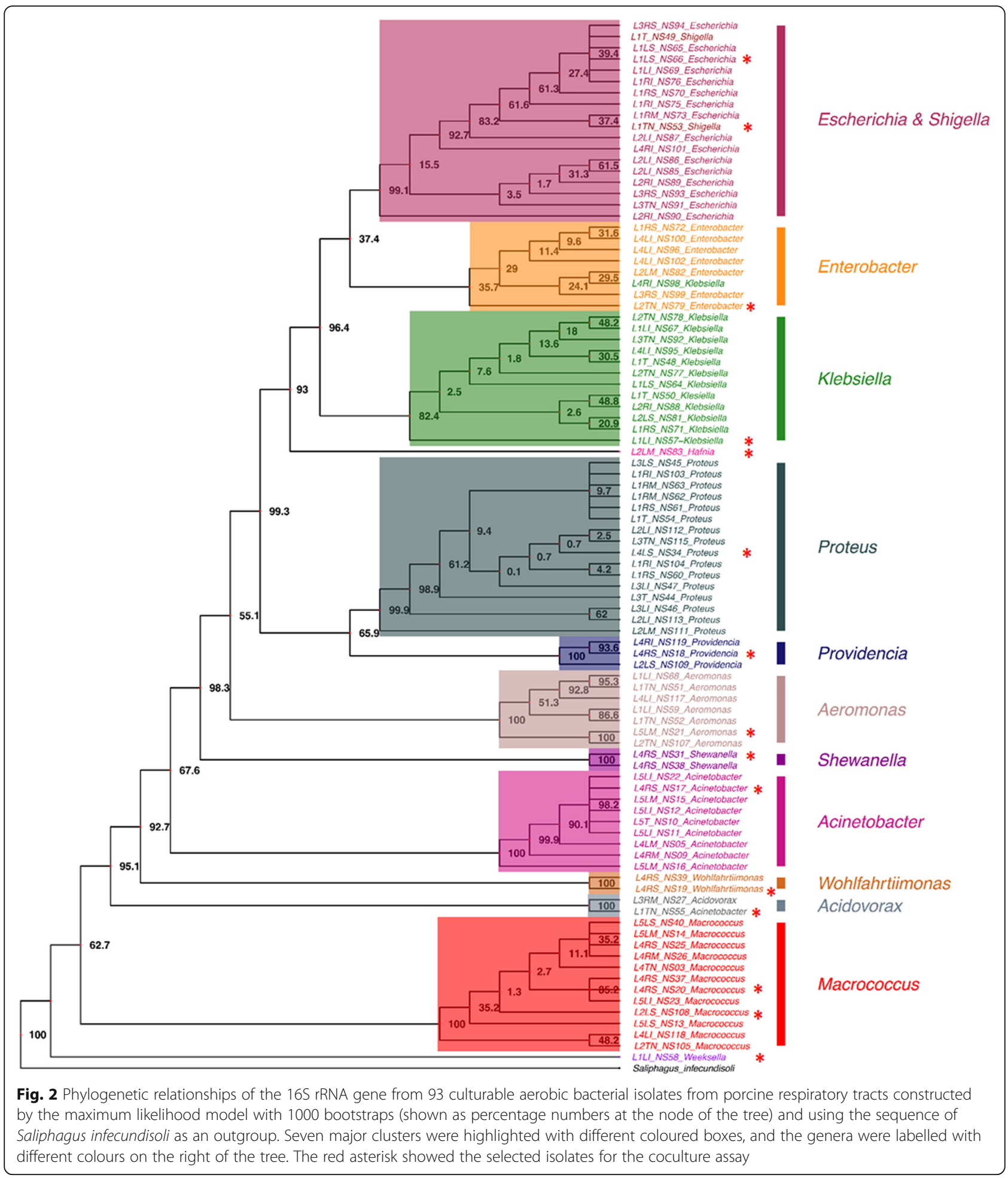

with a prolonged lag phase. The media of five bacterial samples (Klebsiella, Shewanella, Acidovorax, Enterobacter, and Hafnia) only inhibited the growth of P. multocida. The media of both P. multocida strains similarly inhibited Aeromonas, Wohlfahrtiimonas, Shewanella, Acidovorax, Macrococcus, Acinetobacter,
Providencia, and Weeksella as well as themselves. The media of the remaining four samples (Weeksella, Wohlfahrtiimonas, Aeromonas, and Macrococcus G5) had different effects on the tested bacteria. Some conditioned media could promote bacterial growth compared to the control. For example, Weeksella grew 


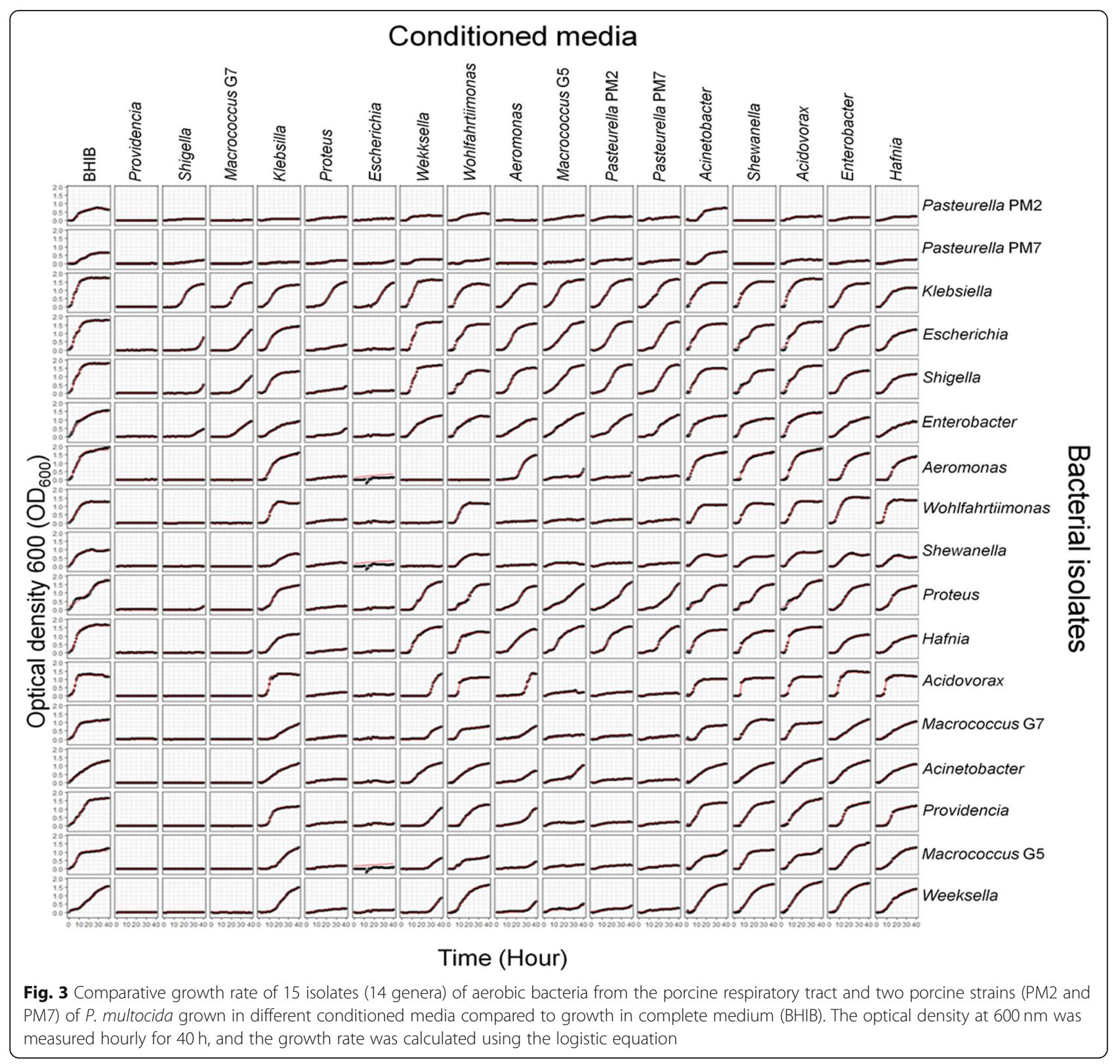

better in the conditioned media of five bacterial samples (Acinetobacter, Wohlfahrtiimonas, Shewanella, Acidovorax, and Enterobacter).

\section{Interaction between the porcine strains of Pasteurella multocida and the selected aerobic bacteria from the porcine respiratory tracts}

This study measured bacterial interactions using the interaction parameter $\varepsilon$, which was calculated from the $\log$ ratio of maximum growth yield in the conditioned medium compared with that in the unconditioned medium. Pairwise interactions between 17 bacterial isolates revealed that most of the interactions (220 interactions) were negative interactions $(\varepsilon<0$ and the interaction scores of the colour scale between orange and pink in Fig. 4). All negative interactions (-/-) were observed when growing the isolates in the conditioned media from Escherichia, Macrococcus, Pasteurella, Proteus, Providencia, Shigella, and Weeksella. Strong negative interactions (59 interactions, $\varepsilon<-1$ ) were observed in the conditioned media of Providencia (17 interactions), Macrococcus G5 (12 interactions), Escherichia (11 interactions), Shigella (11 interactions), and Weeksella (3 interactions), and four interactions were observed in the media of Aeromonas, Klebsiella, and Wohlfahrtiimonas. All spent media had a $\mathrm{pH}$ between 5.0-7.3, which was lower than the $\mathrm{pH}$ of the reference medium BHIB (7.4) (top dendrogram in Fig. 4). Conditioned media from 


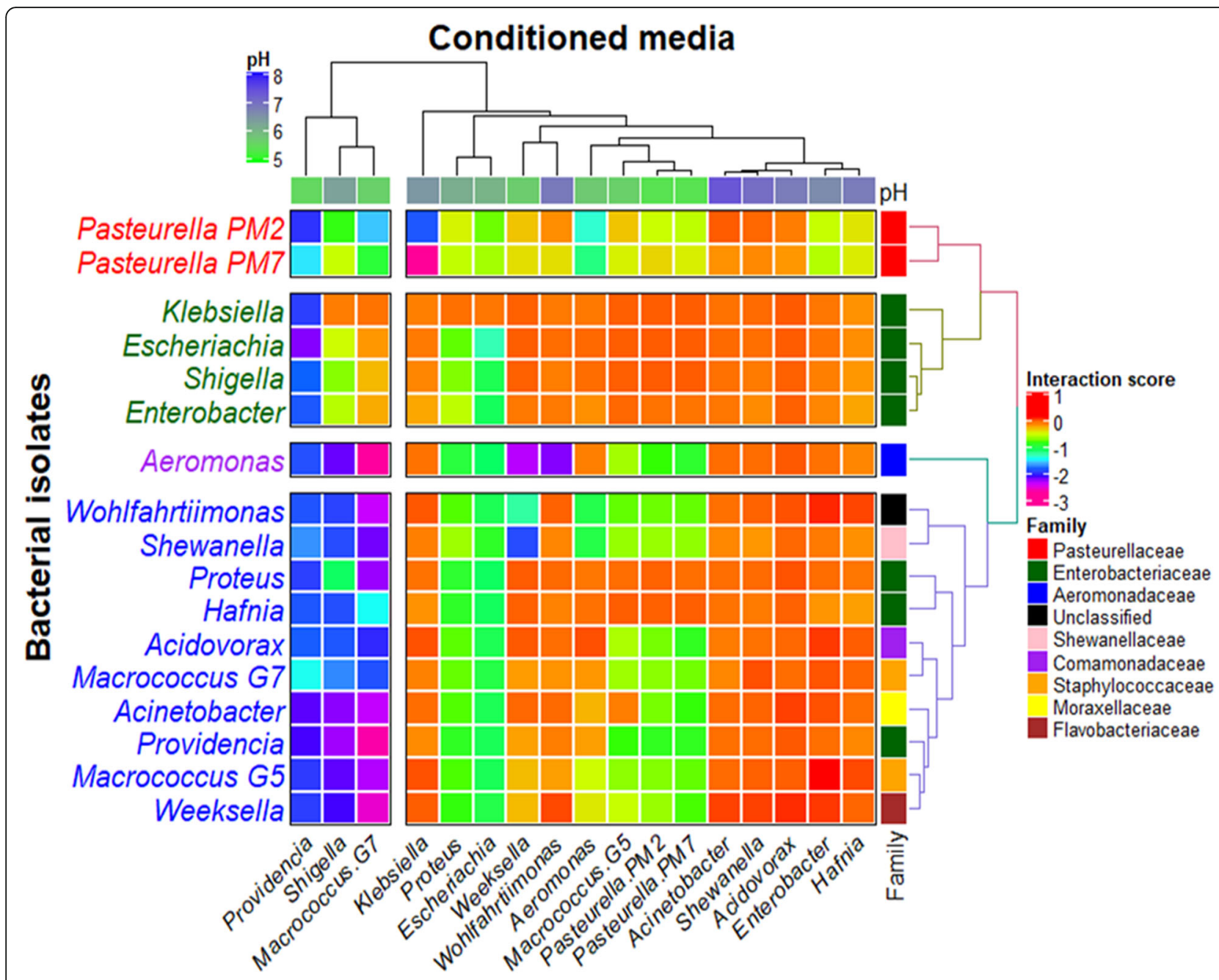

Fig. 4 Interactions between 15 selected bacterial isolates from 14 genera with two porcine strains (PM2 and PM) of $P$. multocida. The interactions were examined by a coculturing assay in conditioned media. The interaction score $(\varepsilon)$ represented the interaction between bacterial isolates: a positive score for a positive interaction and a negative score for a negative interaction. The interaction scores were clustered and labelled by the $\mathrm{pH}$ value of the media (horizontal rows) and bacterial family (vertical columns)

four bacteria (Aeromonas, Klebsiella, Macrococcus G5, and Providencia) had a strong negative effect $(\varepsilon<-1)$ on P. multocida growth and the medium of Klebsiella showed the most substantial impact $(\varepsilon=-1.8$ and -2.6$)$. Notably, the medium of Providencia ( $\mathrm{pH} 5.5$ ) had a strong negative interaction with all tested isolates, including itself. The low $\mathrm{pH}$ (5.4) of the media from the two P. multocida strains resulted in mild to moderate negative interactions with the other tested bacteria. The interaction patterns of $P$. multocida with these 17 conditioned media were separated from those of other bacterial samples (as shown in the right dendrogram) similar to the second cluster of four isolates from the Enterobacteriaceae family and the third cluster of ten samples. Seventeen mild positive interactions $(+/+$, $0<\varepsilon<0.1$ ) were observed with the media of Acidovorax, Acinetobacter, Aeromonas, Enterobacter, Hafnia, Klebsiella, Shewanella, and Wohlfahrtiimonas. Six of these interactions
(Acidovorax, Acinetobacter, Enterobacter, Hafnia, Shewanella, and Wohlfahrtiimonas) observed in spent media with $\mathrm{pH}$ values between $6.5-7.3$, which were close to the $\mathrm{pH}$ of BHIB. Weeksella had positive interactions in five conditioned media, Acidovorax, Acinetobacter, Enterobacter, Shewanella, and Wohlfahrtiimonas, which was the highest number among the media (highlighted in red in Fig. 4). The conditioned medium of Enterobacter supported the highest number of positive interactions with five bacteria, which included Acinetobacter, Acidovorax, Macrococcus G7, Wohlfahrtiimonas, and Weeksella. A high proportion of mild negative interactions (88 interactions, $0>\varepsilon>-0.1$ ) was observed from bacteria grown in nearly all media, except Providencia and Shigella. These mild positive and negative interactions could be classified as neutral interactions. However, strong positive interactions were not observed in this study. 

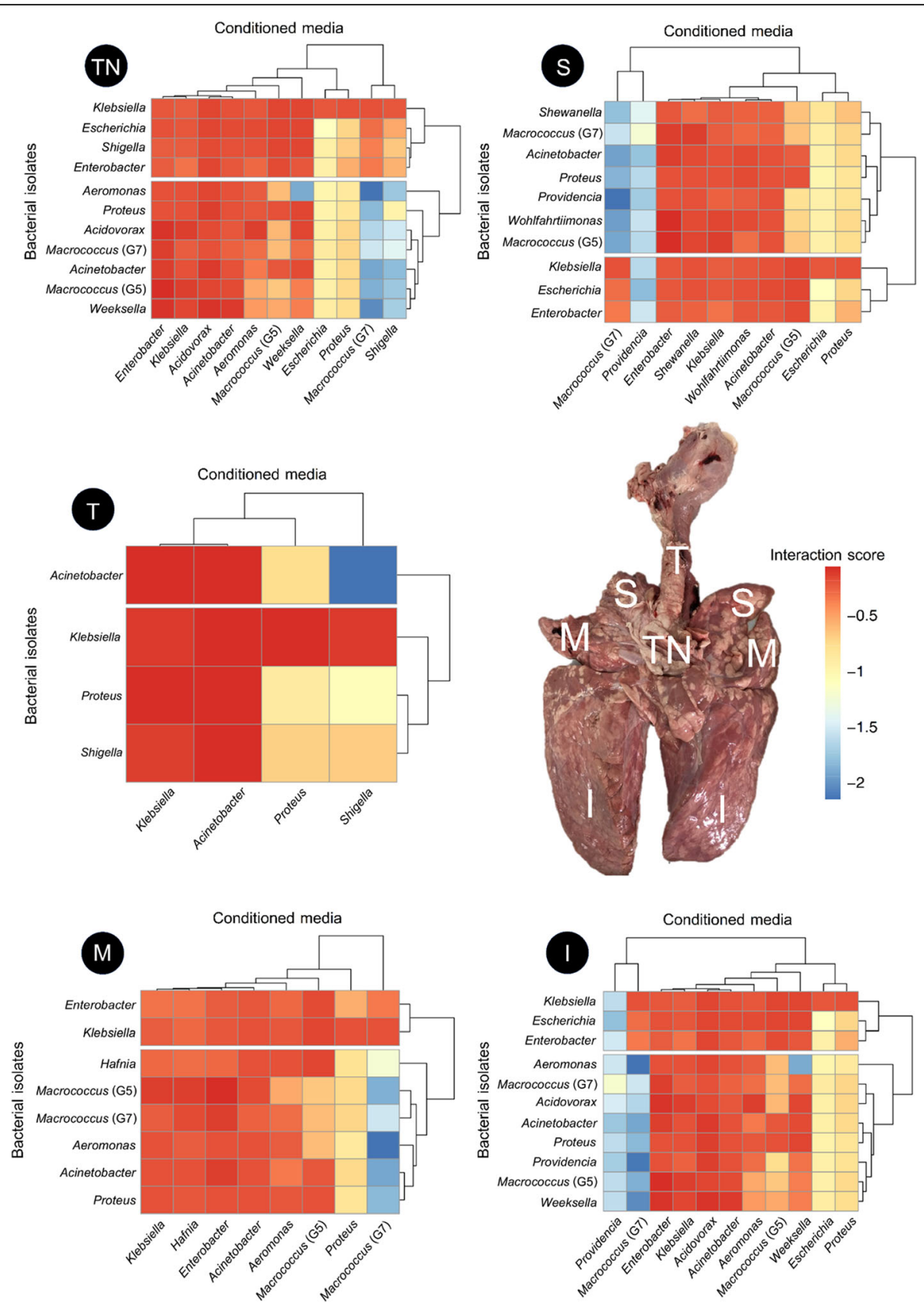

Fig. 5 Bacterial interaction values $(\varepsilon)$ clustered by locations of the porcine respiratory tracts. T, trachea; TN, tracheobronchial lymph node; S, apical lobe; M, cardiac lobe; and I, diaphragmatic lobe of the left and right lungs. A positive interaction score represented a positive interaction (+/+), and a negative interaction score represented a negative interaction (+/-). The top dendrogram shows the conditioned media clustering, and the right dendrogram shows the genera clustering

By comparing the interactions between bacterial isolates found within the same locations of the porcine respiratory tract displayed in Fig. 1, results in Fig. 5 showed that the trachea $(\mathrm{T})$ had the least number of bacterial genera (4 genera) and had Shigella as a strong negative influencer. Macrococcus G7 and Shigella had substantial negative impacts on the others in the tracheobronchial lymph node (TN), while Acidovorax,
Acinetobacter, Enterobacter, and Klebsiella provided positive support to some bacteria in this group. For the apical, cardiac, and diaphragmatic lobes of the porcine lung, Macrococcus G7 was the major bacteria that had a strong negative interaction with the others, except for the apical lobe, in which Providencia also exerted a negative effect. These three lobes of the lung shared five common bacteria with mild negative or positive 
interactions (Acinetobacter, Enterobacter, Klebsiella, Macrococcus, and Proteus), whereas Shewanella and Wohlfahrtiimonas were unique to the apical lobe, Hafnia was unique to the cardiac lobe, and Weeksella was unique to the diaphragmatic lobe.

\section{Discussion}

The porcine respiratory tract has a large mucosal surface area suitable for the colonization of several bacteria, including pathogens [30]. Our study focused on bacterial isolates from porcine respiratory tracts that were culturable and able to grow under aerobic conditions due to the ease of culture and handling so that the isolates could be used for initial coculture experiments to examine their interactions with opportunistic/pathogenic bacteria such as P. multocida. From 116 isolates, our study identified 14 bacterial genera, which included four aerobic (Acidovorax, Acinetobacter, Weeksella, and Wohlfahrtiimonas) and ten facultative anaerobic (Aeromonas, Escherichia, Enterobacter, Hafnia, Klebsiella, Macrococcus, Proteus, Providencia, Shewanella, and Shigella) bacterial genera belonging to seven families (Aeromonadaceae, Comamonadaceae, Enterobacteriaceae, Flavobacteriaceae, Moraxellaceae, Shewanellaceae, and Staphylococcaceae) under three phyla (Proteobacteria, Firmicutes, and Bacteroidetes) from the respiratory tracts of healthy pigs. The isolation of Proteobacteria, Firmicutes, and Bacteroidetes could be due to their abundance in the porcine respiratory tract as previously shown from the metagenomic studies of the nasal, oropharyngeal, and lung microbiota [6-8, 31-35]. Some of these 14 bacterial genera were reported in similar locations of the porcine respiratory tract [5-8, 31-40]. Acinetobacter, Aeromonas, Escherichia, Enterobacter, Klebsiella, Proteus, and Shigella were found in porcine nasal cavity, oropharynx, trachea, lymph node, and lung [5, 6, 8, 31-37, 40]. However, only Acinetobacter, Klebsiella, and Proteus were isolated from the trachea, lymph node, and lung in the present study (Fig. 1). Aeromonas and Enterobacter were isolated from lung and lymph node whereas Shigella was found in the lymph node and trachea, and Escherichia was only found in the lung. The isolation of Weeksella and Shewanella in the porcine lung agreed with the studies of Correa-Fiz et al. [5], Siqueira et al. [6], and Huang et al. [8]. Our study identified Providencia in lung and Macrococcus in lymph node and lung consistent with Mann et al. study [32]. Some discrepancies were also observed when compared the present results to other previous studies. Our study additionally found Hafnia, Macrococcus, Providencia, and Wohlfahrtiimonas in the porcine lung, Acidovorax and Weeksella in the lymph node (Additional file 2).

Possible reasons for these discrepancies could be due to different farming environments (area, temperature, and feed) and pig intrinsic factors (genetics, tissue types, age, and sex). For example, comparative nasal microbiota of healthy piglets from farms in the UK and Spain, which had different farm environments shared at least ten bacterial genera [5]. The identification of Acinetobacter, Klebsiella, and Weeksella in our study was consistent with their results, but only Klebsiella and Weeksella were reported as members of the core nasal microbiota. When compared to the study of oropharyngeal microbiota from healthy piglets in China [7], Streptococcus and Lactobacillus were the core microbiota in the oropharynx of these healthy piglets consistent with the studies in the UK and Spain [5]. This agreement showed that the nasal and oropharynx of the piglets shared more common bacterial genera compared to the tracheal isolates of the mature pigs in the present study. Our study found five bacteria (Acinetobacter, Enterobacter, Klebsiella, Macrococcus, and Proteus) to be the core aerobic bacteria inside the lungs, which was also different from a metagenomic study of the microbiota inside the lungs of healthy pigs in Brazil [6]. Their common microbiota included the families Mycoplasmataceae, Bradyrhizobiaceae, and Flavobacteriaceae, whereas only Aeromonas, Escherichia, and Weeksella were shared with the present study. As the nasal cavity, oropharynx, trachea, and lung are connected parts of the porcine respiratory tract for continuous passage of air and exudate, the aerobic and facultative aerobic bacteria could also colonize multiple locations along the tract. For instance, Acinetobacter and Klebsiella were found in trachea, lung, and lymph node of the present study and in the nasal cavity as reported by Correa-Fiz et al. (2016) [5]. Moreover, the bacterial isolation from three different lobes of the porcine lungs in our study showed some variations in the bacterial genera (Fig. 5), suggesting that the future bacterial sampling of this organ must consider these differences between lobes of the lung. The microbiota of sac and glandlike tissues might be maintained, and the changes may be limited better than the hollow tract with the mucosal surface exposed to the air space within the respiratory tracts. For example, Lowe et al. [35] studied the microbial community inside the tonsils of healthy pigs using culturedependent and culture-independent methods by sequencing 16S rRNA clone libraries. They were able to identify common bacteria (Actinobacillus, Enterobacter, Klebsiella, Pasteurella, Proteus, and Providencia) from porcine tonsils by both methods, and the results were similar to our work. Although our results covered a subset of the bacterial community in porcine respiratory tracts as previously determined by metagenomics studies, the study successfully narrowed and selected particular groups of aerobically grown bacteria from the diverse community to do the coculture assay and shed light on their interactions with $P$. multocida, which might not be easily assessed by wholegenome shotgun metagenomics. 
Opportunistic bacteria, e.g., Pasteurella, Haemophilus, and Actinobacillus, were also abundant in the nasal, oropharynx, and tonsil of healthy pigs but were not isolated under aerobic conditions in this study, which seemed to be a limitation of the bacterial isolation method using the selective media compared to the metagenomics approach which was not required prior bacterial culture $[5,7,34]$. These in vitro-cultured bacterial isolates were good candidates for the coculture assay to examine possible forms of interactions before investigating more complex bacterial interactions in the in vivo or in vivolike experiments. Most bacterial interactions in this study were negative interactions, which represented the competitive need of these bacteria to share resources and spaces [41]. Certain gram-negative bacterial isolates (Providencia, Shigella, Escherichia, and Proteus) in this study yielded conditioned media that strongly inhibited the growth of other bacteria and had similar low $\mathrm{pH}$ compared to the fresh BHIB as shown in Fig. 4. These suggested the release of chemicals or the outgrowth of one bacterium, the isolate used to prepared the conditioned medium, would prevent the growth of the other bacteria later inoculated in this medium. The review by Mattingly and Emonet [42] explained complex bacterial chemotaxis behaviours from the growth on the agar plate by the competition between the nutrient-attractive rapid-growing strains and the slow-growing strains which were non-nutrient attractive. The fast-growing ones would replace (competitive exclusion) or dominate (coexisting) by limiting the growth of the low-performing ones. Harrison et al. [43] examined a coculture between siderophore-producing Staphylococcus aureus and Pseudomonas aeruginosa. These authors found that the absence of free iron induced the production of siderophore by $S$. aureus and increased number of the nonproducing cheater $P$. aeruginosa which would lyse $S$. aureus cells for the irons. Moreover, our coculture assay showed that conditioned media from aerobic bacterial isolates from 13 genera could inhibit the growth of P. multocida. The counteract phenomenon had never been reported between $P$. multocida and these 13 bacterial isolates. As previously described in Harrison et al. [43] and Hibbing et al. [41], the conditioned media of these bacterial isolates could lack certain required nutrients for the growth of $P$. multocida such as irons which might be mostly spent during the preparation of the condition media. If $P$. multocida is directly cocultured with these 13 isolates, it might be out-competed and been excluded from the mixture. However, static liquid coculture or biofilm condition could provide multiple niches for these bacteria to compete and perhaps coexist after several generations (Hibbing et al., 2010) [41].

During the preparation of the conditioned media, bacteria multiplied and spent nutrients in the media, so the conditioned media would have fewer nutrients and plenty of metabolites. Normal growth of bacteria in the conditioned media might imply that these bacteria could co-inhabit the same environment (positive and mild negative interactions), while those affected by scarce nutrients, metabolites, and unsuitable $\mathrm{pH}$ would not be able to thrive together. The bacterial competition also involves several molecular mechanisms. For example, Streptomyces could inhibit antibiotic production by other bacterial competitors to increase its antibiotic production [44]. Barger et al. [45] found that Streptomyces secreted a combination of metabolites and enzymes to degrade colonies and cause cellular lysis of Bacillus subtilis. Competition could cause disproportional populations in the bacterial community and may alter functional relationships in that ecosystem [46]. These interactions could also change the growth conditions of bacteria in the community, increasing or decreasing community complexity [47]. Aside from the effect of bacterial secretion, the rise of one bacterial population could decrease resource availability for another species in the microbiome system. The results of this study also showed that P. multocida had negative interactions with several bacteria and that their conditioned media also inhibited the growth of many bacterial isolates. However, P. multocida could not begin its log phase in almost all conditioned media. This pathogen might have to compete and control the growth of the normal flora bacteria to initiate their multiplication. Competition could also occur within the same bacterial population as in the case of Providencia, suggesting a process to control the population size and initiate spreading to neighbouring areas. The condition that enhances the growth of the normal flora community would provide an inhibitory effect on the pathogens. Therefore, the conditioned media in this study, particularly those with strong negative effect, have to be further characterized to identify key molecules and mechanisms that could control the P. multocida population within the community. In addition to the negative interactions, mild positive interactions were detected for some pairs. Cooperation between microorganisms was reported in a study by Deng and Wang [25], who compared the growth, metabolic activity and enzyme production between pure and mixed cultures in glucose and lignocellulose media. They found that cooperation was common in the lignocellulose media, which promoted positive interactions and synergistic growth. Glucose media promoted negative interactions and competition between organisms in mixed cultures. A study by de Vos et al. [29] also showed that the interaction between bacteria in conditioned media increased bacterial 
tolerance to antibiotics, and positive interactions were observed under non-antibiotic conditions.

\section{Conclusions}

One hundred and sixteen bacterial isolates were collected from five porcine respiratory tracts, and 93 isolates were phylogenetically classified into fourteen genera based on $16 \mathrm{~S}$ rRNA sequences. The coculture of 15 representative isolates and two strains of $P$. multocida showed a majority of negative interactions with a few cooperative/positive interactions. All conditioned media, except those of Acinetobacter, could inhibit P. multocida growth. Conversely, the conditioned media of $P$. multocida also inhibited the growth of eight isolates plus themselves. Thus, this study proposed the possibility of using the molecules in conditioned media to control P. multocida growth and further in vivo-like experiments would be examined to understand the inhibitory mechanism better.

\section{Methods}

\section{Bacterial isolation from porcine respiratory tracts}

Five porcine respiratory tracts were collected from slaughterhouses in Nakhon Pathom and Ratchaburi provinces, Thailand, via the assistance of D.V.M. Pichai Joipang from B.F. Feed Co., Ltd. Bacterial samples were isolated from eight different parts of the respiratory tract, i.e., trachea $(\mathrm{T})$, tracheobronchial lymph node $(\mathrm{TN})$, apical lobe $(\mathrm{S})$, cardiac lobe $(\mathrm{M})$ and the diaphragmatic lobe (I) of both the left and right lungs (LXL and LXR, X was the respiratory tract number). Sample sizes and positions were the same for all five respiratory tracts. The samples were spread on tryptose agar supplemented with $5 \%$ sheep blood and McConkey agar and then incubated aerobically overnight at $37^{\circ} \mathrm{C}$. Colony morphology was observed, and distinct colonies were selected for further subculture on tryptose blood agar. After incubating aerobically overnight at $37^{\circ} \mathrm{C}$, a single colony was picked and subcultured until the pure isolate was obtained. The pure isolate was smeared on a glass slide and checked for purity and bacterial cell morphology by Gram staining and microscopic observation. Extraction of genomic DNA from the pure isolates was performed using the GF-1 bacterial DNA extraction kit (Vivantis, Malaysia), and the genomic DNA was stored in $50 \%$ glycerol with brain and heart infusion broth (BHIB) at $-80^{\circ} \mathrm{C}$ until use.

\section{Bacterial identification by $16 \mathrm{~S}$ rRNA nucleotide sequencing}

Bacterial genera and species were identified by PCR amplification of the $16 \mathrm{~S}$ rRNA gene using SR-FWD (5'AGAGTTTGATYMTGGC-3') and SR-REV (5'-GYTA CCTTGTTACGACTT-3') as forward and reverse primers, respectively [48]. PCR was carried out in a final volume of $20 \mu \mathrm{L}$ containing $2 \mu \mathrm{L}$ of DNA template, $0.4 \mu \mathrm{L}$ of Taq DNA polymerase (Vivantis, Malaysia), $2 \mu \mathrm{L}$ for each of $2 \mu \mathrm{M}$ SR-FWD and SR-REV primers, $0.6 \mu \mathrm{L}$ of $50 \mathrm{mM} \mathrm{MgCl}, 2 \mu \mathrm{L}$ of $2 \mathrm{mM}$ dNTPs (Vivantis, Malaysia), $2 \mu \mathrm{L}$ of 10X Buffer A (Vivantis, Malaysia) and $9 \mu \mathrm{L}$ of distilled water. PCRs were initially denatured at $95^{\circ} \mathrm{C}$ for $5 \mathrm{~min}$, followed by 35 cycles of denaturation at $95^{\circ} \mathrm{C}$ for $45 \mathrm{~s}$, annealing at $50{ }^{\circ} \mathrm{C}$ for $45 \mathrm{~s}$, extension at $72{ }^{\circ} \mathrm{C}$ for $1.35 \mathrm{~min}$, and a final extension at $72^{\circ} \mathrm{C}$ for 5 min using a thermal cycler (Bio-Rad Laboratory Inc., Germany). PCR products were quantified and checked for quality using a NanoDrop 2000 (Thermo Scientific, Germany) before separating on 1\% agarose gel electrophoresis and visualizing under a UV transilluminator (Bio-Rad, United States). The PCR products were purified using the GF-1 AmbiClean kit (Vivantis, Malaysia) and subjected to Sanger nucleotide sequencing (Macrogen, Korea).

Selection of representative bacterial isolates by $16 \mathrm{~S}$ rRNA sequence analysis and phylogenetic reconstruction

The obtained nucleotide sequences of the $16 \mathrm{~S}$ rRNA gene were trimmed and merged by using the BioEdit program version 7.0.5.3 [49]. The sequences were searched against the NCBI nucleotide database using the blastn program [50] to identify closet bacterial species. The identification was decided based on the Blast query score, e-value equal to 0 , and percentage of sequence identity greater than or equal to $99 \%$. As many bacterial isolates were examined and certain isolates belonged to the same genus and species, representative isolates of these bacteria were selected by the following steps. The $16 \mathrm{~S}$ rDNA sequences of bacterial isolates belonging to the same genus were multiple aligned by using the clustalW algorithm in the BioEdit program version 7.0.5.3 $[49,51]$. If the percentage of sequence identity was more than $95 \%$, the sequences were classified into the same genera. If the identity was lower than $95 \%$, the sequences were considered as different genera. The phylogenetic relationship of these $16 \mathrm{~S}$ rDNA sequences was reconstructed from the aligned sequences based on the maximum likelihood algorithm and Jukes-Cantor substitution model with 1000 bootstrap iterations using the phangorn package in $\mathrm{R}[52,53]$. The phylogenetic tree was visualized by the ggtree package in $R$ [54]. The phylogenetic data were used to select the bacterial isolates for the coculture assay. At least one isolate representing the same genus was chosen from the cluster and used to prepare conditioned media for the coculture assay.

\section{Preparation of the conditioned media for the coculture assay}

The conditioned medium was spent medium from the culture of a bacterial isolate. All selected bacterial 
isolates were revived on tryptose blood agar and incubated overnight at $37^{\circ} \mathrm{C}$ before subculture into $40 \mathrm{~mL}$ of BHIB and incubation for $48 \mathrm{~h}$ at $37^{\circ} \mathrm{C}$ and $180 \mathrm{rpm}$. Bacterial cells were pelleted by centrifugation at $4800 \mathrm{x} g$ at room temperature for $15 \mathrm{~min}$. The supernatant medium was filtered by a $0.2 \mu \mathrm{m}$ polyethersulfone (PES) membrane filter (Whatman, United Kingdom) and a $50 \mathrm{~mL}$ syringe (Nipro, United States). The $\mathrm{pH}$ of all conditioned media was measured using a $\mathrm{pH}$ meter (AZ Instrument Corp., Taiwan), and the conditioned media were stored at $4{ }^{\circ} \mathrm{C}$ until use.

\section{Coculture assay and bacterial growth measurement}

Two porcine strains of $P$. multocida (capsular types A (PM7) and D (PM2) isolated from pneumonia pigs in Thailand) and the selected isolates of culturable aerobic bacteria were revived on tryptose blood agar and incubated overnight at $37^{\circ} \mathrm{C}$ before subculture into $1 \mathrm{~mL}$ of BHIB. The coculture assay began by adding $200 \mu \mathrm{L}$ of the conditioned medium into the nontreated transparent flat-bottom 96-well plate followed by inoculating $0.2 \mu \mathrm{L}$ of the overnight bacterial culture. Each bacterial isolate was grown in conditioned media from all chosen isolates. The coculture was incubated at $37^{\circ} \mathrm{C}$ and $180 \mathrm{rpm}$ for $40 \mathrm{~h}$. The optical density at $600 \mathrm{~nm}\left(\mathrm{OD}_{600}\right)$ was measured every hour using a microplate spectrophotometer (PowerWave 340, BioTek, United States), and each condition was performed in triplicate. The bacterial growth rate was calculated using the following logistic equation:

$$
N_{t}=\frac{K}{1+\left(\frac{K-N_{0}}{N_{0}}\right) e^{-r t}}
$$

where $\mathrm{N}_{\mathrm{t}}$ represents the population size at time $\mathrm{t}$, and $\mathrm{N}_{0}$ is the population size at the beginning of the growth curve. The maximum population size in the particular environment was limited to the carrying capacity parameter $\mathrm{K}$. The $\mathrm{OD}_{600}$ values from each condition were fit into this logistic equation to generate the growth curve model by using the SummarizeGrowth function of the Growthcurver package in $\mathrm{R}$ [55]. The growth curves were plotted and compared using the ggplot function of the ggplot2 package to visualize the effect of conditioned media on different bacterial isolates [56].

\section{Determination of the bacterial interactions from the coculture assays}

The definition of the bacterial interaction in this study was adjusted from the study of de Vos et al. [29]. The bacterial interaction was expressed as an interaction parameter $\varepsilon$, described in the following equation:

$$
\varepsilon=\log \left(N_{c} / N_{u}\right)
$$

where $N_{c}$ is the growth yield in the conditioned medium, and $N_{u}$ is the growth yield in the reference medium or fresh BHIB. The growth yield was defined by the average of the four highest $\mathrm{OD}_{600}$ values from the growth curve. The mean of the triplicate maximum growth values in each condition was used to calculate the interaction parameter. A positive $\varepsilon$ value $(\varepsilon>0)$ means that the growth yield in the conditioned medium was higher than that of the reference, indicating the positive or cooperative interaction $(+/+)$ of the two bacterial isolates. A negative or competitive interaction $(-/-)$ corresponds to a negative $\varepsilon$ value $(\varepsilon<0)$. The $\varepsilon \leq-1$ represents strong negative interaction, whereas the $-1<\varepsilon \leq-0.1$ represents moderate negative interaction and $-0.1<\varepsilon<0$ for mild negative interaction. Similarly, $\varepsilon \geq 1$ shows strong positive interaction, whereas the $1>\varepsilon \geq 0.1$ shows moderate positive interaction and $0.1>\varepsilon>0$ for mild positive interaction. The mild negative and positive interactions would be considered as neutral interaction. These parameters were then used to explain the interactions between $P$. multocida and the selected bacterial isolates.

\section{Supplementary Information}

The online version contains supplementary material available at https://doi. org/10.1186/s12866-020-02071-4.

Additional file 1. Details of bacterial isolates. Characteristics and identification of 116 bacterial isolates obtained from the porcine respiratory tracts. Bacterial identification was done by 165 rRNA seqeunce analysis.

Additional file 2. Comparison with other published studies. Comparison of bacterial genera isolated from the porcine respiratory tract in other published studies compared to our study.

\section{Acknowledgements}

The authors would like to thank the Computational Biomodelling Laboratory for Agricultural Science and Technology (CBLAST), Faculty of Science, Kasetsart University for supporting the data analysis and B.F. Feed Co., Ltd. for supporting the sample collection.

\section{Authors' contributions}

$\mathrm{NH}$ carried out bacterial isolation, identification and interaction studies and data analysis. NH, PC, and TE planned and designed the experiments as well as performed data analysis and discussion. NH drafted the manuscript; TE edited and revised the manuscript. All authors have read and approved the manuscript.

\section{Funding}

The research was financially supported by the Science Achievement Scholarship of Thailand (SAST), the National Research Council of Thailand (NRCT), and the Interdisciplinary Graduate Program in Bioscience, Faculty of Science, Kasetsart University, Thailand for the bacterial isolation and identification, co-culture assays, analysis of the bacterial interaction data, and the manuscript publication.

\section{Availability of data and materials}

All data generated or analysed during this study are included in this published article and its supplementary information files.

Ethics approval and consent to participate Not applicable. 


\section{Consent for publication}

Not applicable.

\section{Competing interests}

No competing interests.

\section{Author details}

Interdisciplinary Graduate Program in Bioscience, Faculty of Science, Kasetsart University, Bangkok 10900, Thailand. ${ }^{2}$ Computational Biomodelling Laboratory for Agricultural Science and Technology, Kasetsart University, Bangkok 10900, Thailand. ${ }^{3}$ Department of Zoology, Faculty of Science, Kasetsart University, Bangkok 10900, Thailand. ${ }^{4}$ Department of Genetics, Faculty of Science, Kasetsart University, Bangkok 10900, Thailand. ${ }^{5}$ Omics Center for Agriculture, Bioresources, Food, and Health, Kasetsart University (OmiKU), Bangkok 10900, Thailand.

Received: 18 June 2020 Accepted: 15 December 2020 Published online: 09 January 2021

\section{References}

1. Bals R, Hiemstra PS. Innate immunity in the lung: how epithelial cells fight against respiratory pathogens. Eur Respir J. 2004;23(2):327-33.

2. O'Boyle N, Sutherland E, Berry CC, Davies RL. Temporal dynamics of ovine airway epithelial cell differentiation at an air-liquid interface. PLoS One. 2017:12(7):e0181583.

3. Holt HR, Alarcon P, Velasova M, Pfeiffer DU, Wieland B. BPEX pig health scheme: a useful monitoring system for respiratory disease control in pig farms? BMC Vet Res. 2011;7:82

4. Opriessnig T, Giménez-Lirola LG, Halbur PG. Polymicrobial respiratory disease in pigs. Anim Health Res Rev. 2011;12(2):133-48.

5. Correa-Fiz F, Fraile L, Aragon V. Piglet nasal microbiota at weaning may influence the development of Glasser's disease during the rearing period. BMC Genomics. 2016;17:404.

6. Siqueira FM, Pérez-Wohlfeil E, Carvalho FM, Trelles O, Schrank IS Vasconcelos ATR, Zaha A. Microbiome overview in swine lungs. PLoS One. 2017:12(7):e0181503.

7. Wang Q, Cai R, Huang A, Wang X, Qu W, Shi L, Li C, Yan H. Comparison of oropharyngeal microbiota in healthy piglets and piglets with respiratory disease. Front Microbiol. 2018;9:3218

8. Huang T, Zhang M, Tong X, Chen J, Yan G, Fang S, Guo Y, Yang B, Xiao S, Chen $\mathrm{C}$, et al. Microbial communities in swine lungs and their association with lung lesions. Microb Biotechnol. 2019;12(2):289-304

9. Boyce JD, Harper M, Wilkie IW, Adler B. Pasteurella. In: Gyles CL, Prescott JF, Songer JG, Thoen CO, editors. Pathogenesis of Bacterial Infections of Animals. lowa: Blackwell Publishing; 2010. p. 325-46.

10. Loera-Muro A, Ramírez-Castillo FY. Porcine Respiratory Disease Complex and Biofilms. J Bacteriol Parasitol. 2015:6:247.

11. Brockmeier SL, Halbur PG, Thacker EL. Porcine respiratory disease complex. In: Brogden KA, Guthmiller JM, editors. Polymicrobial diseases. Washington (DC): ASM Press; 2002.

12. Lainson FA, Aitchison KD, Donachie W, Thomson JR. Typing of Pasteurella multocida isolated from pigs with and without porcine dermatitis and nephropathy syndrome. J Clin Microbiol. 2002;40(2):588-93.

13. Register KB, Brockmeier SL, de Jong MF, Pijoan C. Pasteurellosis. In: Zimmerman JJ, Kaeeiker LA, Ramirez A, Schwartz KJ, Stevenson GW, editors. Diseases of Swine. 10th ed. Amess IA: Wiley-Blackwell; 2012. p. 798-810.

14. Wilson BA, Ho M. Pasteurella multocida: from zoonosis to cellular microbiology. Clin Microbiol Rev. 2013:26(3):631-55.

15. Liu H, Zhao Z, Xi X, Xue Q, Long T, Xue Y. Occurrence of Pasteurella multocida among pigs with respiratory disease in China between 2011 and 2015. Ir Vet J. 2017;70:2.

16. JXd OF, MAZ M, Rebelatto R, AMD A, CLA P, Klein CS, DESN B, Morés N. Pasteurella multocida type $\mathrm{A}$ as the primary agent of pneumonia and septicaemia in pigs. Pesqui Vet Bras. 2015;35(8):716-24.

17. Pedersen KB, Barfod K. The aetiological significance of Bordetella bronchiseptica and Pasteurella multocida in atrophic rhinitis of swine. Nordisk Vet Med. 1981:33(12):513-22.

18. Dugal F, Belanger $M$, Jacques $M$. Enhanced adherence of Pasteurella multocida to porcine tracheal rings preinfected with Bordetella bronchiseptica. Can J Vet Res. 1992;56(3):260-4.
19. Chanter N, Magyar T, Rutter JM. Interactions between Bordetella bronchiseptica and toxigenic Pasteurella multocida in strophic rhinitis of pigs. Res Vet Sci. 1989;47(1):48-53.

20. Nakai T, Kume K, Yoshikawa H, Oyamada T, Yoshikawa T. Adherence of Pasteurella multicida or Bordetella bronchiseptica to the swine nasal epithelial cell in vitro. Infect Immun. 1988:56:234-40.

21. Sudaryatma PE, Nakamura K, Mekata H, Sekiguchi S, Kubo M, Kobayashi I, Subangkit M, Goto Y, Okabayashi T. Bovine respiratory syncytial virus infection enhances Pasteurella multocida adherence on respiratory epithelial cells. Vet Microbiol. 2018;220:33-8.

22. Pors SE, Hansen MS, Bisgaard M, Jensen HE, Iburg TM. Immunohistochemical study of porcine lung lesions associated with Pasteurella multocida. Vet J. 2013;197(2):483-8.

23. Bartkiene E, Lele V, Starkute V, Zavistanaviciute P, Zokaityte E, Varinauskaite I, Pileckaite G, Paskeviciute L, Rutkauskaite G, Kanaporis T, et al. Plants and Lactic Acid Bacteria Combination for New Antimicrobial and Antioxidant Properties Product Development in a Sustainable Manner. Foods. 2020;9(4):433.

24. DeLeon S, Clinton A, Fowler H, Everett J, Horswill AR, Rumbaugh KP. Synergistic interactions of Pseudomonas aeruginosa and Staphylococcus aureus in an in vitro wound model. Infect Immun. 2014;82(11):4718-28.

25. Deng YJ, Wang SY. Synergistic growth in bacteria depends on substrate complexity. J Microbiol. 2016;54(1):23-30.

26. Zhang C, Wu X, Wu Y, Li J, An H, Zhang T. Enhancement of dicarboximide fungicide degradation by two bacterial cocultures of Providencia stuartii JD and Brevundimonas naejangsanensis J3. J Hazard Mater. 2021;403:123888.

27. Heyse J, Buysschaert B, Props R, Rubbens P, Skirtach AG, Waegeman W, Boon N. Coculturing Bacteria Leads to Reduced Phenotypic Heterogeneities. Appl Environ Microbiol. 2019;85(8):e02814-18.

28. Mathieu-Denoncourt A, Letendre C, Auger JP, Segura M, Aragon V, Lacouture S, Gottschalk M. Limited interactions between Streptococcus suis and Haemophilus parasuis in in vitro co-infection studies. Pathogens. 2018 7(1):7

29. de Vos MGJ, Zagorski M, McNally A, Bollenbach T. Interaction networks, ecological stability, and collective antibiotic tolerance in polymicrobial infections. Proc Natl Acad Sci U S A. 2017;114(40):10666-71.

30. Louten J. Virus Transmission and Epidemiology. In: Louten J, editor. Essential Human Virology. London: Elsevier; 2016. p. 71-92. https://doi.org/10.1016/ B978-0-12-800947-5.00005-3.

31. Dosen R, Prodanov J, Milanov D, Stojanov I, Pusic I. The bacterial infections of respiratory tract of swine. Biotechnol Anim Husbandry. 2007;23(5-6-2): 237-43.

32. Mann E, Dzieciol M, Pinior B, Neubauer V, Metzler-Zebeli BU, Wagner M, Schmitz-Esser S. High diversity of viable bacteria isolated from lymph nodes of slaughter pigs and its possible impacts for food safety. J Appl Microbiol. 2015:119(5):1420-32.

33. Mann E, Pinior B, Wetzels SU, Metzler-Zebeli BU, Wagner M, Schmitz-Esser S The metabolically active bacterial microbiome of tonsils and mandibular lymph nodes of slaughter pigs. Front Microbiol. 2015;6:1362.

34. Lowe BA, Marsh TL, Isaacs-Cosgrove N, Kirkwood RN, Kiupel M, Mulks MH. Defining the "core microbiome" of the microbial communities in the tonsils of healthy pigs. BMC Microbiol. 2012;12(12):20.

35. Lowe BA, Marsh TL, Isaacs-Cosgrove N, Kirkwood RN, Kiupel M, Mulks MH. Microbial communities in the tonsils of healthy pigs. Vet Microbiol. 2011; 147(3-4):346-57.

36. Zhao G, Zhang L, Li C, Zhao J, Liu N, Li Y, Wang J, Liu L. Identification of enterobacteria in viscera of pigs afflicted with porcine reproductive and respiratory syndrome and other viral co-infections. Microb Pathog. 2020;147: 104385

37. Stoltz DA, Meyerholz DK, Pezzulo AA, Ramachandran S, Rogan MP, Davis GJ, Hanfland RA, Wohlford-Lenane C, Dohrn CL, Bartlett JA, et al. Cystic fibrosis pigs develop lung disease and exhibit defective bacterial eradication at birth. Sci Transl Med. 2010:2(29):29ra31.

38. Risco D, Pedro F-L, Jesús MC, José MB-M, Waldo LG-J, Remigio M, Pilar G, Javier HDM, Luis G, Alfredo G. Isolation of Aeromonas hydrophila in the respiratory tract of wild boar: pathologic implications. J Zoo Wildl Med. 2013:44(4):1090-3.

39. Slifierz MJ, Friendship RM, Weese JS. Longitudinal study of the early-life fecal and nasal microbiotas of the domestic pig. BMC Microbiol. 2015:15(1):184

40. Weese J, Slifierz M, Jalali M, Friendship R. Evaluation of the nasal microbiota in slaughter-age pigs and the impact on nasal methicillin-resistant Staphylococcus aureus (MRSA) carriage. BMC Vet Res. 2014;10(1):69. 
41. Hibbing ME, Fuqua C, Parsek MR, Peterson SB. Bacterial competition: surviving and thriving in the microbial jungle. Nat Rev Microbiol. 2010; 8(1):15-25.

42. Mattingly $\mathrm{H}$, Emonet $\mathrm{T}$. A rule from bacteria to balance growth and expansion. Nature. 2019;575(7784):602-3.

43. Harrison F, Paul J, Massey RC, Buckling A. Interspecific competition and siderophore-mediated cooperation in Pseudomonas aeruginosa. ISME J. 2008;2(1):49-55.

44. Abrudan MI, Smakman F, Grimbergen AJ, Westhoff S, Miller EL, van Wezel GP, Rozen DE. Socially mediated induction and suppression of antibiosis during bacterial coexistence. Proc Natl Acad Sci U S A. 2015;112(35):11054-9.

45. Barger SR, Hoefler BC, Cubillos-Ruiz A, Russell WK, Russell DH, Straight PD. Imaging secondary metabolism of Streptomyces sp. Mg1 during cellular lysis and colony degradation of competing Bacillus subtilis. Antonie Van Leeuwenhoek. 2012;102(3):435-45.

46. Becker J, Eisenhauer N, Scheu S, Jousset A. Increasing antagonistic interactions cause bacterial communities to collapse at high diversity. Ecol Lett. 2012;15(5):468-74.

47. Morin M, Pierce EC, Dutton RJ. Changes in the genetic requirements for microbial interactions with increasing community complexity. Elife. 2018;7: e37072.

48. Dey S, Singh VP, Kumar AA, Sharma B, Srivastava SK, Singh N. Comparative sequence analysis of $16 \mathrm{~S}$ rRNA gene of Pasteurella multocida serogroup B isolates from different animal species. Res Vet Sci. 2007:83(1):1-4.

49. Hall TA. BioEdit: a user-friendly biological sequence alignment editor and analysis program for windows 95/98/nt. Nucleic Acids Symp. 1999:41:95-8.

50. Altschul SF, Gish W, Miller W, Myers EW, Lipman DJ. Basic local alignment search tool. J Mol Biol. 1990;215(3):403-10.

51. Thompson JD, Higgins DG, Gibson TJ. CLUSTAL W: improving the sensitivity of progressive multiple sequence alignment through sequence weighting, position-specific gap penalties and weight matrix choice. Nucleic Acids Res. 1994;22(22):4673-80.

52. Felsenstein J. Evolutionary trees from DNA sequences: a maximum likelihood approach. J Mol Evol. 1981;17(6):368-76.

53. Penny D, Hendy M. Estimating the reliability of evolutionary trees. Mol Biol Evol. 1986;3(5):403-17.

54. Guangchuang Y, Smith DK, Zhu H, Guan Y, Lam TT-Y, Mclnerny G. ggtree: an R package for visualization and annotation of phylogenetic trees with their covariates and other associated data. Methods Ecol Evol. 2017:8(1):28-36.

55. Sprouffske K, Wagner A. Growthcurver: an R package for obtaining interpretable metrics from microbial growth curves. BMC Bioinformatics. 2016;17(1):172

56. Wickham H. ggplot2: elegant graphics for data analysis. New York: SpringerVerlag; 2016.

\section{Publisher's Note}

Springer Nature remains neutral with regard to jurisdictional claims in published maps and institutional affiliations.

Ready to submit your research? Choose BMC and benefit from:

- fast, convenient online submission

- thorough peer review by experienced researchers in your field

- rapid publication on acceptance

- support for research data, including large and complex data types

- gold Open Access which fosters wider collaboration and increased citations

- maximum visibility for your research: over $100 \mathrm{M}$ website views per year

At $\mathrm{BMC}$, research is always in progress.

Learn more biomedcentral.com/submissions 